\title{
INFLUÊNCIA DO PODER NAS REDES DE NEGÓCIO - ANÁLISE DA EVOLUÇÃO DA TEORIA
}

\author{
Luis Oliveira* \\ luis.oliveira@usp.br \\ Mário Sacomano Neto** \\ msacomano@ufscar.br \\ João Maurício Gama Boaventura* \\ jboaventura@usp.br \\ * Universidade de São Paulo - São Paulo, SP / Brasil \\ ** Universidade Federal de São Carlos - São Carlos, SP / Brasil \\ Editada pela Escola de Administração da Universidade Federal do Rio Grande do Sul.
}

http://dx.doi.org/10.1590/1413-2311.025162016.62022

Recebido em 03/02/2016

Aprovado em 12/05/2016

Disponibilizado em 06/06/2016

Avaliado pelo sistema "double blind review"

Revista Eletrônica de Administração

Editora-chefe: Aurora Zen

ISSN 1413-2311 (versão "on line")

Periodicidade: Quadrimestral

Sistema requerido: Adobe Acrobat Reader

\section{RESUMO}

Esta pesquisa busca compreender como as relações de poder são abordadas na literatura de organizações, por meio de uma revisão sistemática da literatura utilizando a base de dados Thomson Reuters (ISI) Web of Science. Embora a literatura de relações interorganizacionais reconheça o papel crítico do poder, uma sucessão de teorias com diferentes perspectivas empíricas, teóricas e metodológicas demonstra que ainda há uma falta de consenso a respeito de quais atores detêm o poder nestes contextos. São questões de pesquisa: Como os recentes estudos em redes de negócio abordam o poder? Quais as implicações dos principais paradigmas teóricos utilizados? A análise das publicações selecionadas aponta para a multiplicidade de perspectivas teóricas na abordagem do poder na literatura e indica um aumento do interesse no tema ao longo do tempo. As principais perspectivas teóricas identificadas são a Teoria da Dependência de Recursos; a Teoria da Troca em Redes; e a Teoria de Custos de Transação. Essas teorias são analisadas sob o quadro teórico e um conjunto de proposições conceituais é definido, de modo a avaliar as implicações dos paradigmas associados a cada corrente teórica.

Palavras-Chave: Poder; Redes de Negócios; Paradigmas. 


\title{
INFLUÊNCIA DO PODER NAS REDES DE NEGÓCIO - ANÁLISE DA EVOLUÇÃO
}

DA TEORIA

\section{INFLUENCE OF POWER IN BUSINESS NETWORKS - ANALYSIS OF THE THEORY'S EVOLUTION}

\begin{abstract}
This research aims to understand how power relations are addressed in organizations literature, with a systematic literature review of the Thomson Reuters (ISI) Web of Science Database. Although the literature on interorganizational relationships recognizes the critical role of power, a succession of theories with different empirical, theoretical and methodological perspectives demonstrates that there is still a lack of consensus about which actors hold the power in these contexts. The research questions are: How do recent studies in business networks deal with power? What are the implications of the main theoretical paradigms used? Analysis of the selected publications points to the multiplicity of theoretical perspectives on power in the literature and indicates an increased interest in the subject over time. The major theoretical perspectives identified are the Resource Dependency Theory; the Network Exchange Theory; and the Transaction Costs Theory. These theories are discussed under the theoretical framework and a set of conceptual propositions is defined in order to assess the implications of the paradigms associated with each theoretical stream.
\end{abstract}

Keywords: Power; Business Networks; Paradigms.

\section{INFLUENCIA DEL PODER EN REDES EMPRESARIALES - ANÁLISIS DE LA EVOLUCIÓN DE LA TEORÍA}

\begin{abstract}
RESUMEN
Esta investigación busca comprender cómo se abordan las relaciones de poder en la literatura de organizaciones, mediante una revisión sistemática de la literatura utilizando la base de datos Thomson Reuters (ISI) Web of Science. Aunque la literatura sobre relaciones interorganizacionales reconozca el papel crítico del poder, una sucesíon de teorías con variadas perspectivas empíricas, teóricas y metodológicas demuestra que todavía hay una falta de consenso sobre cuales actores detienen el poder en estos contextos. Las preguntas de investigación son: ¿Cómo estudios recientes en redes empresariales tratan del poder? ¿Cuáles son las implicaciones de los principales paradigmas teóricos utilizados? El análisis de las publicaciones seleccionadas indica la multiplicidad de perspectivas teóricas em el enfoque de poder en la literatura, así como un aumento del interés en el tema al paso del tiempo. Las principales perspectivas teóricas identificadas son la Teoría de la Dependencia de Recursos; la Teoría del Intercambio; y la Teoría de los Costes de Transacción. Estas teorías se discuten con base en el marco teórico y un conjunto de proposiciones conceptuales se define a fin de evaluar las consecuencias de los paradigmas asociados a cada corriente teórica.
\end{abstract}

Palabras Clave: Poder; Redes de Negocios; Paradigmas. 


\section{INTRODUÇÃO}

Esta pesquisa busca compreender como as relações de poder são abordadas na literatura de organizações, utilizando a base de dados Thomson Reuters (ISI) Web of Science. A pesquisa utiliza a revisão sistemática como forma de colete e reflexão sobre os dados bibliométricos. Por meio da localização e análise estruturada da produção acadêmica prévia, a revisão sistemática permite o mapeamento e a análise do estado da literatura (DENYER; TRANFIELD, 2009; TRANFIELD; DENYER; SMART, 2003).

A visão de empresas como unidades isoladas competindo entre si no mercado é cada vez mais inadequada à dinâmica dos negócios (GULATI; NOHRIA; ZAHEER, 2000; HINGLEY, 2005; RICCOBONO; BRUCCOLERI; PERRONE, 2013). De forma alternativa, é reconhecido que as empresas estão imersas em redes de negócios definidas por um conjunto diverso de relacionamentos que podem ultrapassar as fronteiras de indústrias e países (GULATI; NOHRIA; ZAHEER, 2000) manifestando, por meio deste arranjo, traços de competitividade que não existem em cada um dos negócios de forma isolada (ZACCARELLI et al., 2008). A percepção do arranjo empresarial como um processo coletivo não é algo elementar mas advém da evolução dos níveis de fidelização entre os negócios e da consequente intensificação dos relacionamentos (ZACCARELLI et al., 2008).

A estabilidade e o sucesso desses relacionamentos no longo prazo são usualmente associadas às condições de poder e mutualidade existentes, ainda que visões distintas se desenvolvam a respeito do papel e da relevância do poder nessas relações (BELAYA; HANF, 2012; BENGTSSON; KOCK, 2000; BLOIS, 2010; HINGLEY, 2005). Embora a literatura de relações interorganizacionais reconheça o papel crítico do poder, uma sucessão de teorias com diferentes perspectivas empíricas, teóricas e metodológicas demonstra que ainda há uma falta de consenso a respeito de quais atores detêm o poder nestes contextos (MEEHAN; WRIGHT, 2012; RODRIGUEZ et al., 2007). A tipologia priorizada para as discussões também é alvo de um conjunto de críticas que defendem a necessidade de um modelo mais adequado à dinâmica das relações de negócio atualmente estudadas (MEEHAN; WRIGHT, 2012).

Em uma rede há uma considerável variedade de conexões entre múltiplos níveis e no modo como tais conexões são mobilizadas (SMITH-DOERR; POWELL, 2004). Dado que a literatura de negócios passa a incorporar fatores contextuais na análise destas conexões, afastando-se de uma ótica determinística derivada essencialmente dos agentes em contato 


\section{INFLUÊNCIA DO PODER NAS REDES DE NEGÓCIO - ANÁLISE DA EVOLUÇÃO} DA TEORIA

(HINGLEY, 2005; MEEHAN; WRIGHT, 2012), considera-se que isso resulte em impactos sobre como as relações de poder são abordadas na literatura. Assim, este estudo aborda o poder como algo determinante para compreensão das organizações e redes.

São questões de pesquisa deste estudo: Como os recentes estudos em redes de negócio abordam o poder? Quais as implicações dos principais paradigmas teóricos utilizados? Como ponto de partida, entende-se que prevalecerão nos estudos do poder em redes de negócios as principais teorias associadas aos paradigmas da própria literatura de redes de negócios. A existência de uma perspectiva preferencial acarreta impactos para o desenvolvimento do campo, dado que perspectivas distintas carregam premissas e limites de validade próprios (MORGAN, 1980).

$\mathrm{Na}$ literatura de redes de negócios, os principais paradigmas correspondem às abordagens relacional e estrutural. A adoção de um ou outro quadro teórico privilegia uma determinada operacionalização do construto, o que pode tornar tendenciosa a abordagem de um tema ainda controverso. Tal consideração abre espaço para reflexões adicionais sobre as implicações desses paradigmas, recordando que a maior parte da pesquisa sobre poder tem se apoiado de modo genérico na tipologia proposta por French e Raven (1959).

Este trabalho está dividido em seis seções, a contar desta introdução. A próxima seção apresenta o referencial teórico, com um quatro conceitual para a análise do poder nas organizações. A seção seguinte detalha a metodologia utilizada na pesquisa, qual seja uma revisão sistemática da literatura, e uma caracterização dos artigos filtrados é encontrada na seção subsequente. Em sequência, os artigos selecionados são discutidos à luz do quadro conceitual delimitado no referencial teórico. A seção final oferece uma conclusão para o trabalho, esclarecendo as limitações existentes e sugerindo direções para pesquisas futuras.

\section{REFERENCIAL TEÓRICO}

\subsubsection{Paradigmas nas teorias sociais}

O uso de diferentes lentes para a análise de um tema é resultado da adoção de diferentes perspectivas por parte dos teóricos que se propoem a essa investigação. Segundo Morgan (1980) indica, os paradigmas são os elementos centrais da visão de mundo dos teóricos nas teorias sociais. Ao nível dos paradigmas, é possível reconhecer que teorias 
distintas compartilham pressupostos, mas também se diferenciam em várias proporções. $\mathrm{O}$ autor detalha duas verticais que refletem quatro quadrantes, onde poderiam ser classificadas as teorias sociais (Figura 1).

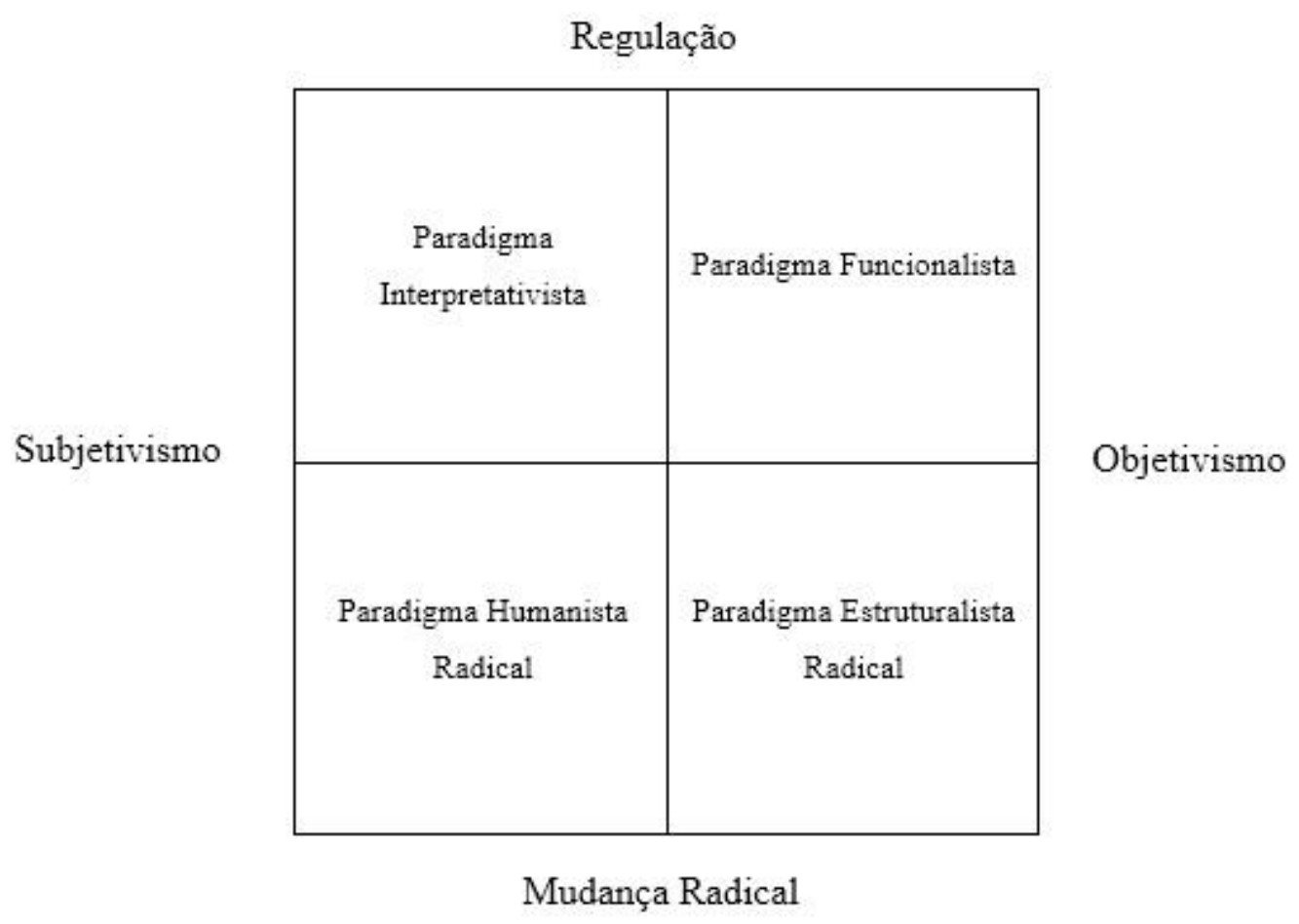

Figura 1 - Paradigmas da Teoria Social

Adaptado de: Morgan (1980)

Em um eixo, são contrapostas as perspectivas objetiva e subjetiva. Na primeira a sociedade tem uma existência concreta e independente, onde o pesquisador é um observador, ao passo que na segunda ele está nela imerso e ela existe através da sua interpretação. No segundo eixo, opõem-se a visão de regulação e a de mudança radical. Essas visões diferem com relação ao modo como se encaram as regras e padrões sociais: a primeira sustenta a compreensão de uma ordem social que é combatida e considerada opressora pela segunda.

Cada um desses grupos representa lentes fundamentalmente diferentes para a análise das organizações e favorece o desenvolvimento de metáforas igualmente distintas. Morgan (1980) sugere que a comunidade científica tem muito a ganhar dessa percepção, a partir do momento em que se pode buscar complementaridade para a interpretação de um fenômeno em disciplinas distitnas. Ainda assim, críticos acusam seu trabalho de promover disputas e a polarização do campo (CALDAS, 2005).

REAd | Porto Alegre - Edição 83 - N 1 - Janeiro/Abril 2016 - p. 01-25 


\section{INFLUÊNCIA DO PODER NAS REDES DE NEGÓCIO - ANÁLISE DA EVOLUÇÃO DA TEORIA}

Lewis e Grimes (1999) reconhecem na pesquisa multiparadigmática o surgimento de respostas ao apelo daquele autor. Eles identificam essa abordagem em duas formas de estudos. De um lado, os estudos paralelos identificam conflitos e interesses pela contraposição de lentes teóricas distintas. Por outro lado, os estudos sequenciais alimentam estudos subsequentes a partir dos resultados obtidos sob uma determinada perspectiva; a alternância das lentes utilizadas permitem explorar a divergência dos focos e ganhar a consciência de elementos ocultos em uma visão monoparadigmática.

\subsubsection{Evolução das teorias sobre redes}

Segundo Todeva (2006), os estudos de redes de negócios orientam-se segundo três abordagens principais, cada qual carrega um conjunto de paradigmas que se complementam de modo a formar um conjunto coerente de teorias e metodologias para a análise de redes. Convém compreender estas diferentes abordagens, na medida em que suas lentes distintas têm implicações sobre a forma como o poder é discutido. A autora detalha cada uma delas:

- $\quad$ Abordagem Estrutural: Enfatiza a estrutura das redes, fazendo sua análise sob o ponto de vista da posição que os atores nela ocupam e segundo a qual passam a agir. A relação entre estes atores é destituída da sua individualidade: passam a existir apenas conexões que se apresentam em diferentes estados de simetria e força e a interação entre os agentes não pode ser facilmente desagregada em ações de atores individuais. Regras e normas são interpretadas como perfis comportamentais padrão.

- $\quad$ Abordagem Relacional: De modo oposto, os atores são tomados em sua individualidade, com porte, estrutura, experiência e tecnologia que os tornam únicos. Redes paralelas co-existem: redes de atores conectados aos pares; redes de recursos que através deles fluem e redes de atividades interconectadas que manifestam sua participação na cadeia. O dinamismo dessas conexões produz uma análise movida a eventos, uma vez que a intensidade e a polarização das relações são mutáveis. Aos atores humanos é atribuída a concepção de escolhas estratégicas, que dizem respeito a instituições não-humanas.

- $\quad$ Abordagem Cultural: Vai ainda além e dota os atores com a capacidade de agir, interpretar e imaginar as redes. Estas são sistemas vivos e complexos, capazes de evolir modificando sua estrutura e gerenciando seu ambiente interno e externo. A REAd | Porto Alegre - Edição 83 - N 1 - Janeiro/Abril 2016 - p. 01-25 
Luis Oliveira, Mário Sacomano Neto \& João Maurício Gama Boaventura

identificação dos atores-chave é de fundamental interesse, na medida em que seus valores e interesses influenciam o movimento da rede.

\subsubsection{O Poder na literatura de Organizações}

É reconhecido que o poder é um elemento presente nas diferentes formas de relação social (BIERSTEDT, 1950) e que surge em contextos tão variados que dificilmente seriam comparáveis (DAHL, 1957). A maior parte da pesquisa relacionada ao tema tem se apoiado de modo genérico em uma mesma tipologia (MEEHAN; WRIGHT, 2012). Este modelo, proposto por French e Raven (1959), divide em cinco as principais fontes de poder entre dois indivíduos:

- $\quad$ Recompensa: refere-se à percepção que um indivíduo tem de que outro seja capaz de mediar recompensas para ele;

- Coercivo: remete à percepção que um indivíduo tem de que outro seja capaz de mediar punições para ele;

- Legítimo: corresponde à percepção que um indivíduo tem de que outro tenha direitos legítimos de prescrever um comportamento a ele;

- $\quad$ Referente: está baseado na identificação que um indivíduo tenha em relação a outro;

- $\quad$ Especialista: reside na percepção que um indivíduo tem de que outro detenha um conhecimento especial ou especializado.

Os três primeiros são classificados como poderes mediados, uma vez que carregam em sua base a motivação de induzir uma ação, ao contrário dos dois restantes, que residem na identificação e internalização da vontade de cooperar e que por isso não classificados como não mediados (HANDLEY; BENTON, 2012). Algumas críticas com relação a este modelo se referem à capacidade de extrapolar o contexto original da pesquisa, originalmente orientada a indivíduos em uma relação de supervisor e supervisionado no ambiente de trabalho, à potencial indisponibilidade de todas as cinco formas de poder em determinados contextos e na baixa capacidade de explicação das atitudes do supervisor em comparação à sua capacidade de lidar com a cooperação do supervisionado (MEEHAN; WRIGHT, 2012).

Muito embora seja possível reconhecer essa convergência em torno de uma tipologia comum, a evolução dessa literatura acompanha o amadurecimento da própria literatura de 


\section{INFLUÊNCIA DO PODER NAS REDES DE NEGÓCIO - ANÁLISE DA EVOLUÇÃO} DA TEORIA

negócios - que evolui de um foco em transações individuais a uma abordagem de relacionamento. A discussão de poder inter-organizacional passa assim por uma transformação, internalizando elementos como mutualidade, colaboração e confiança (HINGLEY, 2005). Diferentes escolas de pensamento podem ser pontuadas em função da unidade de análise preferencial, esteja ela ao nível das organizações, dos indivíduos ou dos relacionamentos (MEEHAN; WRIGHT, 2012).

Para a literatura de compras e cadeias de suprimentos, o poder é uma propriedade das organizações. Conforme essa abordagem, a performance da relação que se estabelece entre compradores e fornecedores depende do seu alinhamento com as condições de poder em que se encontram (COX et al., 2004; SANDERSON, 2004). Sob certas condições, relacionamentos desalinhados podem ser ajustados para uma melhor eficiência quando há a mudança de comportamento de uma das partes (COX et al., 2004).

Estudos no campo de negociação e correntes de marketing atribuem o poder aos indivíduos. O foco das análises está no indivíduo comprador (BONOMA; JOHNSTON, 1978), que define e analisa situações de compra, para então decidir e agir (WEBSTER, 1972). Um complexo conjunto de objetivos pessoais e organizacionais devem então ser considerados, além de influenciadores externos, restrições variadas e a própria personalidade do indivíduo (BONOMA; JOHNSTON, 1978; WEBSTER, 1972).

Finalmente, há as pesquisas que se preocupam em discutir o poder em termos de relações contextualizadas. Para esta corrente, analisar o poder sem considerar fatores situacionais é assumir uma regularidade inexistente no comportamento humano independentemente da variação cultural (CHENG; SCULLI; CHAN, 2001; HO, 1991). Assim, agentes se comportam e interagem de modo diferente em diferentes situações, com impactos sobre suas fontes de poder disponíveis e como estas são percebidas (HO, 1991).

\section{MÉTODO}

Dada a multiplicidade de concepções para o poder através de diversos campos e a importância que este tema tem demonstrado para a compreensão de fenômenos em redes de negócios, há vantagens na escolha da revisão sistemática como método de pesquisa. Este método é caracterizado pela localização e análise estruturada da produção acadêmica prévia (DENYER; TRANFIELD, 2009). Sua importância para a área de gestão reside na capacidade REAd | Porto Alegre - Edição 83 - N 1 - Janeiro/Abril 2016 - p. 01-25 
de permitir o mapeamento e a análise do estado da literatura (TRANFIELD; DENYER; SMART, 2003).

Denyer e Tranfield (2009) indicam que a condução deste método deve ter em consideração a natureza do problema de pesquisa e o tipo de evidência disponível. Segundo os autores, a aplicação deste método em pesquisas na área de gestão e estudos organizacionais devem ser replicáveis; considerar as melhores evidências disponíveis; permitir análises agregadas; e ser específicos com relação ao algoritmo utilizado.

A fim de lidar com estes pontos, os procedimentos de busca e seleção dos artigos são aqui apresentados em detalhe. Uma pesquisa foi realizada na base de dados Thomson Reuters (ISI) Web of Science, em busca de documentos em que figurassem no Tópico (Título, Resumo ou Palavras-Chave) os termos "business network". As áreas temáticas foram limitadas pela afinidade com o tema a: Management, Business, Operations Research Management Science, Economics, Sociology, Business Finance, Social Sciences Interdisciplinary. Ainda, outro filtro foi empregado para reduzir a amostra aos documentos classificados pela produção no tipo article.

Para focar o estudo nas produções mais atuais da literatura de interesse, foi feito um filtro de data para selecionar artigos mais recentes. O período foi definido de 2003 a 2012, para tomar os últimos dez anos completos de pesquisa, resultando em 3.560 artigos selecionados. Dentre os resultados, foi realizado um novo filtro ao se procurar o termo "power" no grupo de publicações, o que limitou a amostra mais uma vez, para 249 artigos. A análise qualitativa dos títulos e resumos destas publicações permitiu fazer uma nova redução da amostra de artigos, finalizando em 26 documentos.

A seção a seguir faz uma discussão do resultado dessa revisão sistemática. Além da caracterização descritiva e da avaliação crítica dos textos, é explorada a rede de cocitações da pesquisa, produzida com o auxílio das ferramentas BibExcel e NodeXL. 
INFLUÊNCIA DO PODER NAS REDES DE NEGÓCIO - ANÁLISE DA EVOLUÇÃO DA TEORIA

\section{RESULTADOS}

\subsubsection{Análise das Publicações}

\subsubsection{Caracterização dos Dados}

Uma análise da origem dos documentos selecionados mostra que estão pulverizados ao longo de 26 diferentes publicações, sendo que o Industrial Marketing Management é o mais recorrente - com seis artigos. De outro modo, 16 periódicos surgem com apenas um texto cada (Tabela 1). Isso é um indicativo de um baixo nível de convergência entre as diferentes linhas de pesquisa interessadas em discutir o poder em um contexto de redes de negócio.

Por outro lado, o número de publicações por ano (Figura 2) sugere um aumento gradual de interesse no tema ao longo do tempo. A ocorrência de publicações vinha numa tendência de alta até 2007. Após uma breve queda entre os anos de 2008 e 2009, há uma retomada a partir de 2010 e então nova indicação de alta.

Tabela 1 - Periódicos mais frequentes

\begin{tabular}{|c|c|}
\hline Periódico & Artigos \\
\hline Industrial Marketing Management & 6 \\
\hline Futures & 2 \\
\hline Journal of Economic Geography & 2 \\
\hline Outros & 16 \\
\hline
\end{tabular}

Fonte: Elaborado pelos autores 


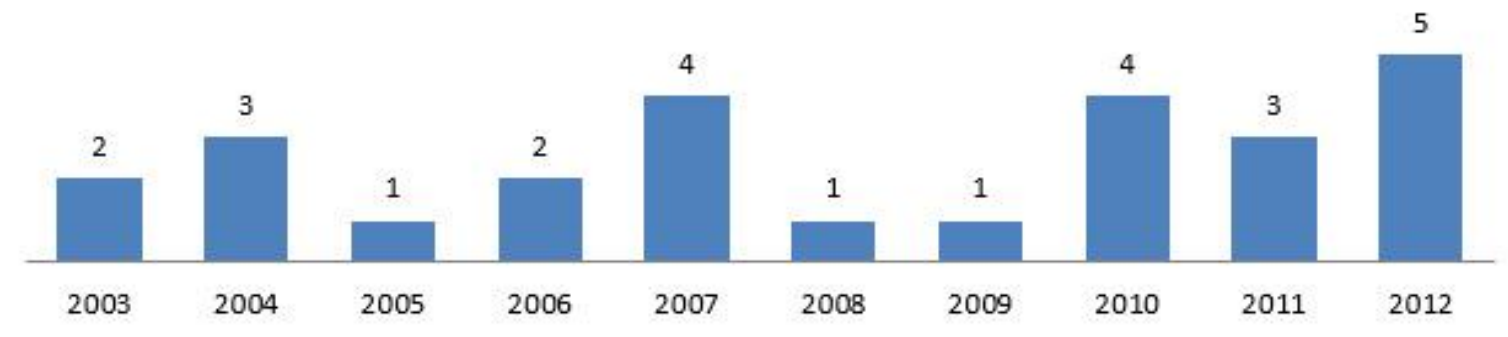

Gráfico1 - Número de publicações por ano na amostra pesquisada

Fonte: Elaborado pelos autores

Conforme Todeva (2006), a literatura de redes de negócios tem sido construída primariamente com o uso de estudos de casos. Seguindo Freese (1980), a construção de um campo teórico tipicamente tem início com dados empiricamente coletados e sistematizados em linguagem comum. A Tabela 2 indica um equilíbrio entre a quantidade de estudos encontrada em cada nível dentre os artigos selecionados, sugerindo uma maior maturidade para o campo de estudo com a construção de teoria e a verificação empírica caminhando juntas.

Tabela 2 - Distribuição dos artigos segundo o delineamento da pesquisa

\begin{tabular}{|c|c|c|c|c|}
\hline $\begin{array}{c}\text { Nível } \\
\text { Delineamento }\end{array}$ & Exploratória & Descritiva & Explicativa & Total \\
\hline Bibliográfica & 5 & 1 & & 6 \\
\hline Documental & 1 & & 1 & 2 \\
\hline Estudo de Campo & & 1 & 1 & 2 \\
\hline Estudo de Caso & 2 & 7 & 3 & 12 \\
\hline Experimento & & & 1 & 1 \\
\hline Simulação & & 1 & & 1 \\
\hline Survey & & 1 & 1 & 2 \\
\hline Total & 8 & 11 & 7 & 26 \\
\hline
\end{tabular}

Fonte: Elaborado pelos autores

De outro modo, existe uma pulverização na escolha do delineamento para os estudos.

Isso pode ser tomado como reflexo da multiplicidade de abordagens existentes para a REAd | Porto Alegre - Edição 83 - Nº 1 - Janeiro/Abril 2016 - p. 01-25 


\section{INFLUÊNCIA DO PODER NAS REDES DE NEGÓCIO - ANÁLISE DA EVOLUÇÃO DA TEORIA}

discussão de poder, conforme exposto anteriormente. $\mathrm{O}$ volume de estudos de caso, destacado dentre os demais formatos, reforça o caráter dinâmico de uma área em expansão. A presença de estudos teóricos bibliográficos em segundo lugar é outro indício de um estágio de maturidade em que passa a ser possível construir sobre um arcabouço de publicações já reconhecidas no meio acadêmico.

\subsubsection{Ocorrência dos tipos de poder e teorias utilizadas}

A Tabela 3 traz a distribuição dos artigos pesquisados conforme a principal teoria neles utilizada. Para esta classificação, os artigos foram avaliados em função dos seus referenciais teóricos para a discussão do poder. Quando a teoria utilizada não estava tão clara, as citações foram pesquisadas para que fosse possível identificar o quadro teórico que fundamentava sua construção.

Tabela 3 - Distribuição dos artigos segundo a teoria fundam
\begin{tabular}{|c|c|}
\hline Teoria & Artigos \\
\hline Dependência de Recursos & 13 \\
\hline Teoria da Troca em Redes & 5 \\
\hline Teoria dos Custos de Transação & 3 \\
\hline Teoria da Agência & 2 \\
\hline Teoria de Campos & 2 \\
\hline Teoria do Evento-Estrutura & 1 \\
\hline Elaborado pelos autores
\end{tabular}

Sob cada uma dessas teorias, o poder pode ser interpretado de formas diferentes. O Quadro 1 a seguir oferece um resumo dessas interpretações: 
Luis Oliveira, Mário Sacomano Neto \& João Maurício Gama Boaventura

Quadro 1 - Abordagem para o poder segundo diferentes teorias

\begin{tabular}{|c|c|c|}
\hline Teoria & Autor(es) & Abordagem para o Poder \\
\hline $\begin{array}{c}\text { Teoria da Dependência } \\
\text { de Recursos }\end{array}$ & Pfeffer e Salancik (1978) & $\begin{array}{c}\text { O poder é baseado no controle de recursos que } \\
\text { são considerados estratégicos }\end{array}$ \\
\hline $\begin{array}{c}\text { Teoria da Troca em } \\
\text { Redes }\end{array}$ & Cook et al. (1983) & $\begin{array}{c}\text { O poder de A sobre B pode ser definido como } \\
\text { a probabilidade de A obter resultados } \\
\text { favoráveis aos custos de B }\end{array}$ \\
\hline $\begin{array}{c}\text { Teoria dos Custos de } \\
\text { Transação }\end{array}$ & Williamson (1981) & $\begin{array}{c}\text { As estruturas de governança (e, portanto, as } \\
\text { relações de poder) variam em função das } \\
\text { condições de incerteza, frequência de troca e } \\
\text { especificidade de ativos }\end{array}$ \\
\hline $\begin{array}{c}\text { Teoria da Agência } \\
\text { Teoria de Campos }\end{array}$ & Jensen e Meckling (1976) & $\begin{array}{c}\text { O conflito de interesses entre o ator principal e } \\
\text { otor agente torna necessários mecanismos de } \\
\text { garantia de poder }\end{array}$ \\
\hline $\begin{array}{c}\text { Teoria do Evento- } \\
\text { Estrutura }\end{array}$ & Fligstein (2007) & $\begin{array}{c}\text { O poder se manifesta na disputa entre grupos } \\
\text { pelo dominio do campo }\end{array}$ \\
\hline Dahl (1957) & $\begin{array}{c}\text { O poder de A sobre B é definido como a } \\
\text { probabilidade de que A consiga que B faça } \\
\text { algo que de outro modo não faria }\end{array}$ \\
\hline
\end{tabular}

Fonte: Elaborado pelos autores

Em função da discussão de poder proposta, os documentos foram avaliados com base na tipologia definida por French e Raven (1959). O resultado dessa classificação aparece na Figura 3. Aqueles artigos que não nomeavam explicitamente um tipo de poder conforme a tipologia indicada foram interpretados de modo a serem nela também classificados.

Dois pontos são notáveis na Figura 3. Primeiramente, a ausência de poder classificado como referente e, em segundo lugar, a predominância de poder coercitivo. Em função da dependência contextual da tipologia e das limitações relacionadas anteriormente, considerouse improdutivo especular as razões por trás destes fatos. Em lugar disso, produziu-se uma nova análise que objetivou investigar as consequências para o campo a partir das escolhas teóricas das publicações selecionadas. 
INFLUÊNCIA DO PODER NAS REDES DE NEGÓCIO - ANÁLISE DA EVOLUÇÃO DA TEORIA

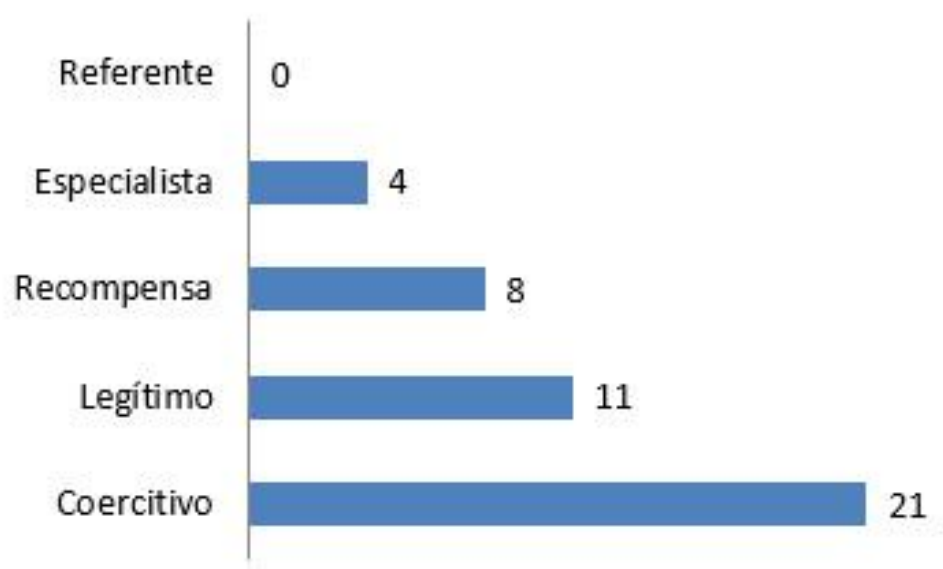

Gráfico 2 - Distribuição dos artigos segundo a tipologia de poder estudada

Fonte: Elaborado pelos autores

Fazendo um cruzamento entre os tipos de poder identificados na Figura 3 e a relação de teorias mencionadas no

Quadro 1, é possível observar a distribuição do uso de diferentes quadros teóricos (Tabela 4). Uma vez que há artigos que discutem mais de um tipo de poder, é natural verificar que a soma dos valores nas linhas da Tabela 4 não corresponde aos números apresentados na Tabela 3.

Percebe-se que as teorias da dependência de recursos e a teoria da troca em redes são aquelas que apresentam uma maior flexibilidade no sentido de figurar na discussão de um maior número de tipos de poder. Ainda, a primeira destas teorias é a mais utilizada com larga vantagem. De modo contrário, as teorias da agência e do evento-estrutura estão limitadas cada um à discussão de apenas dois tipos de poder.

Tabela 4 - Relação entre os tipos de poder e as teorias utilizadas

\begin{tabular}{|c|c|c|c|c|}
\hline Teoria & Especialista & Recompensa & Legítimo & Coercitivo \\
\hline Dependência de Recursos & 2 & 6 & 4 & 12 \\
\hline Teoria da Troca em Redes & 1 & 1 & 1 & 4 \\
\hline Teoria dos Custos de Transação & 1 & & 1 & 2 \\
\hline
\end{tabular}

REAd | Porto Alegre - Edição 83 - N 1 - Janeiro/Abril 2016 - p. 01-25 
Luis Oliveira, Mário Sacomano Neto \& João Maurício Gama Boaventura

\begin{tabular}{|c|c|c|c|c|}
\hline Teoria da Agência & & & 2 & 1 \\
\hline Teoria de Campos & & 1 & 2 & 1 \\
\hline Teoria do Evento-Estrutura & & & 1 & 1 \\
\hline
\end{tabular}

Fonte : Elaborado pelos autores

Uma reflexão acerca do uso preferencial da teoria da dependência de recursos será oferecida na discussão dos resultados. Do mesmo modo, são discutidas as implicações do uso das teorias mais utilizadas (recorrência em ao menos 3 artigos da Tabela 3), considerando o tipo de poder associado e os seus fundamentos de tais teorias. Na análise se buscará oferecer um olhar inicial sobre as raízes praradigmáticas das teorias relacionadas.

\subsubsection{Análise da Rede de Cocitações}

Após uma primeira análise descritiva das publicações filtradas na busca, uma análise da rede de cocitações permite identificar padrões de relacionamento entre os estudos com respeito às suas raízes teóricas. O racional dessa análise indica que referências citada em conjunto constiuirão uma estrutura consensual para os conceitos em um campo. Ainda, é possível avaliar a atualização do campo a partir da comparação da influência de textos seminais com outros, mais recentes.

A Figura 4 apresenta a rede de cocitações da pesquisa produzida com o auxílio das ferramentas BibExcel e NodeXL. Apenas aparecem pares de artigos que tiveram ao menos duas cocitações e os artigos estão representados pelo nome do primeiro autor e seu ano de publicação. O tamanho dos nós é proporcional ao seu grau de centralidade, ao passo que a espessura das linhas é proporcional à frequência de cocitação das referências ligadas. 


\section{INFLUÊNCIA DO PODER NAS REDES DE NEGÓCIO - ANÁLISE DA EVOLUÇÃO} DA TEORIA

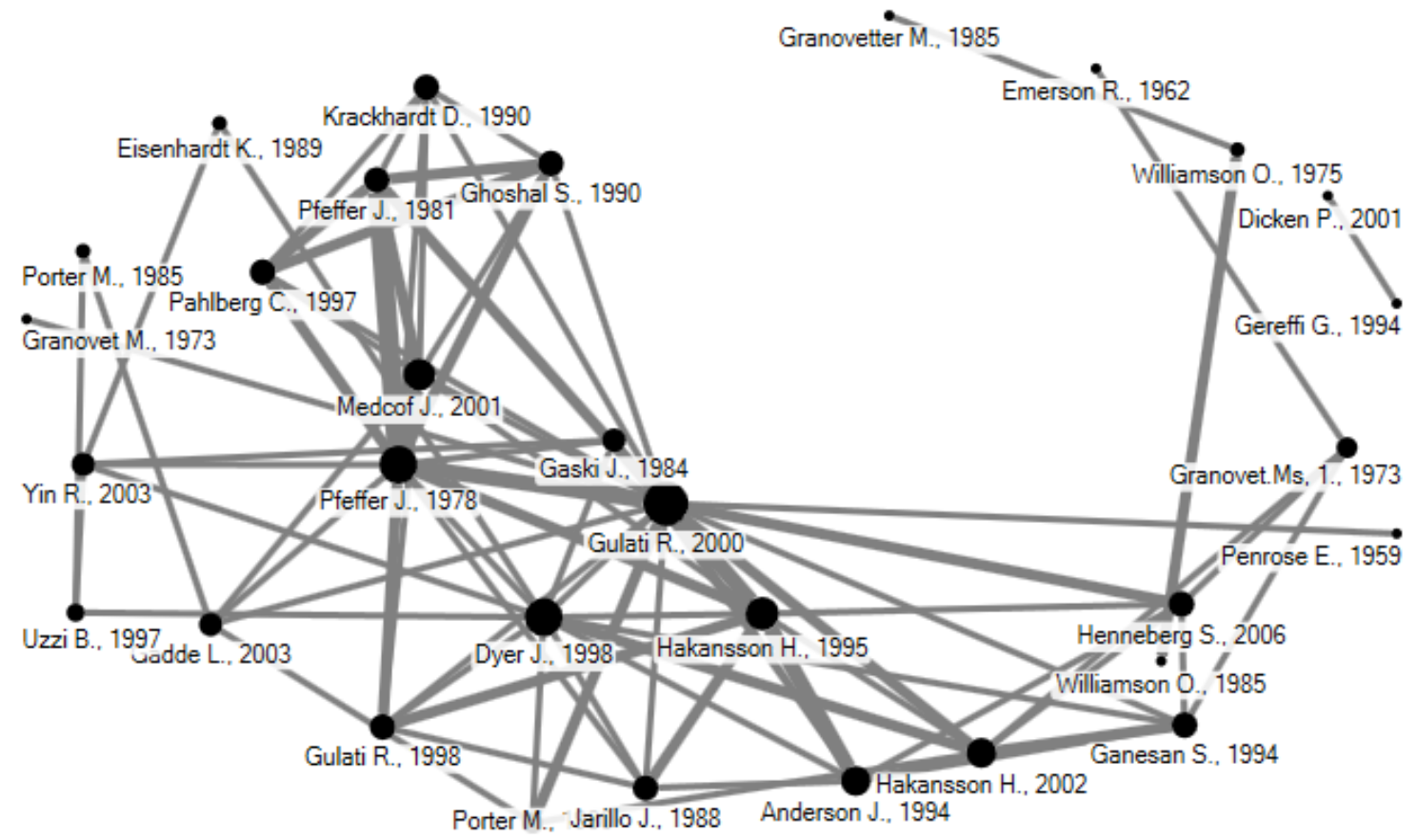

Figura 2 - Rede de Cocitações

Fonte: Elaborado pelos autores

As Tabelas 5 e 6 relacionam as cinco referências com os maiores graus de centralidade e intermediação na rede construída, respectivamente. Nota-se que o artigo de Gulati, Nohria e Zaheer (2000) encabeça ambas as listas de centralidade e intermediaão. Isso indica que essa publicação não apenas possui o maior número de conexões diretas com as demais, mas também é a que mais intermedia conexões entre todos os pares possíveis da rede.

Ainda, vale verificar que os textos de Gulati, Nohria e Zaheer (2000), Pfeffer e Salancik (1978) e Dyer e Singh (1998) figuram nas duas listas em posições de destaque. A presença desses textos em destaque é um indicativo importante para a compreensão da trajetória de atualização do campo e será discutida mais adiante, juntamente com os demais resultados apresentados.

Tabela 5 - Referências com os maiores graus de centralidade

\begin{tabular}{|c|c|}
\hline Referência & Grau de Centralidade \\
\hline Gulati, Nohria e Zaheer (2000) & 18 \\
\hline Pfeffer e Salancik (1978) & 13 \\
\hline Dyer e Singh (1998) & 10 \\
\hline
\end{tabular}

REAd | Porto Alegre - Edição 83 - N 1 - Janeiro/Abril 2016 - p. 01-25 
Luis Oliveira, Mário Sacomano Neto \& João Maurício Gama Boaventura

\begin{tabular}{|c|c|}
\hline Håkansson e Snehota (1995) & 10 \\
\hline Medcof (2001) & 9 \\
\hline
\end{tabular}

Fonte: Elaborado pelos autores

Tabela 6 - Referências com os maiores graus de intermediação

\begin{tabular}{|c|c|}
\hline Referência & Grau de Intermediação \\
\hline Gulati, Nohria e Zaheer (2000) & 113,187 \\
\hline Dyer e Singh (1998) & 59,120 \\
\hline Pfeffer e Salancik (1978) & 34,956 \\
\hline Granovetter (1973) & 24,000 \\
\hline Håkansson e Ford (2002) & 20,916 \\
\hline
\end{tabular}

Fonte : Elaborado pelos autores

\section{DISCUSSÃO DOS RESULTADOS}

\subsubsection{Pilares teóricos na análise do poder}

A maior ocorrência do uso da teoria da dependência de recursos (Tabela 3) demanda uma reflexão particular a respeito das motivações e circunstâncias responsáveis por tal destaque. É possível interpretar este fato de forma razoável sob os dois principais paradigmas de estudo de redes, de forma que em ambos se verá uma tendência ao uso desta teoria.

Primeiramente, sob a abordagem relacional, vale recordar o modelo A-R-A (HÅKANSSON; JOHANSON, 1993 apud TODEVA, 2006), que captura a complexidade desta abordagem. O modelo sobrepõe três redes - uma rede de atores interligados; uma rede de recursos que por eles fluem e uma rede de atividades interconectadas - e três possibilidades de interdependência - de recursos, de atividades e de atores. Discutir o poder embasado particularmente na teoria da dependência de recursos representa assumir um foco sobre a dimensão mais facilmente visualizável e administrável da rede. Isso porque fluxos de recursos ocorrem quando atores trocam produtos ou serviços entre si (PFEFFER; SALANCIK, 1978).

A respeito da abordagem estrutural, é possível recordar que é uma corrente dividida em duas perspectivas: analítico-funcional e analítico-estrutural (TODEVA, 2006). Enquanto a perspectiva analítico-funcional está focada no comportamento das firmas e na dependência de recursos desenvolvida entre elas em função de atividades tipicamente desenvolvidas em 


\section{INFLUÊNCIA DO PODER NAS REDES DE NEGÓCIO - ANÁLISE DA EVOLUÇÃO} DA TEORIA

regimes de cooperação, a perspectiva analítico-estrutural investiga a proximidade entre estas empresas com ênfase nas diferentes formas de imersão social.

Considerando evidências de que tais perspectivas são complementares e que a estrutura influencia a formação de alianças particularmente na presença de motivadores funcionais (CASCIARO, 2003), é compreensível que a dependência de recursos também seja uma teoria fundamental ao discutir o poder com este enquadramento.

A divisão dos textos entre tais pilares fica também evidenciada na avaliação da rede de cocitações construída a partir das referências desses artigos (Figura 4). Três artigos recebem destaque nessa análise, segundo ambas as medidas de centralidade (Tabela 5) e intermediação (Tabela 6). O texto de Pfeffer e Salancik (1978) introduz a visão baseada em recursos, lançando luzes sobre a influência do ambiente externo das organizações sobre seu comportamento. Sua grande relevância na rede de artigos aparece em sintonia com o mapeamento das teorias predominantes.

Nota-se também que Dyer e Singh (1998) e Gulati, Nohria e Zaheer (2000) também são textos com destaque no conjunto de referências e ajudam a compreender a trajetória de atualização do campo teórico. Particularmente, estas publicações aparecem com mais intermediações do que Pfeffer e Salancik (1978). Sua relevância e intermediação podem ser compreendidos uma vez que são artigos que realizaram importantes contribuições teóricas na extensão da pesquisa acadêmica.

Dyer e Singh (1998) oferecem uma contribuição à visão da firma baseada em recursos, sugerindo que os recursos críticos de uma empresa podem ultrapassar suas fronteiras. Os autores chamam a atenção para a relação entre firmas como unidade de análise, podendo ser fonte de diferentes tipos de vantagens competitivas: ativos específicos da relação; rotinas de compartilhamento de conhecimento; recursos/competências complementares; e governança efetiva. Gulati, Nohria e Zaheer (2000), por sua vez; aproxima a visão de redes de negócios e a literatura de estratégia, avaliando que superar a perspectiva da firma como uma entidade independente seria algo especialmente benéfico a cinco áreas-chave: estrutura da indústra; posicionamento na indústria; recursos e competências inimitáveis; custos de contratação e coordenação; e restrições e benefícios dinâmicos da rede. 


\subsubsection{Consequências das principais teorias}

A seguir procura-se interpretar as principais teorias relacionadas na Tabela $3 \mathrm{em}$ função das características dos paradigmas associados. A visão de que uma mesma teoria é utilizada para interpretar mais de um tipo de poder pode ser considerada do ponto de vista da coerência entre os tipos de poder discutidos e os paradigmas associados às lentes teóricas.

As três teorias mais utilizadas são analisadas a seguir em relação ao quadro traçado no referencial teórico. Além disso, algumas proposições conceituais são definidas com base nas reflexões desenvolvidas. Tais proposições conectam as raízes das teorias com seu leque de utilização na explicação dos tipos de poder. O teste dessas proposições não é alvo deste artigo.

\subsubsection{Teoria da Dependência de Recursos}

A teoria da dependência de recursos foi introduzida por Pfeffer e Salancik (1978), aplicando os conceitos de Emerson (1962) ao contexto organizacional. Os autores definem que qualquer evento que dependa de um ou mais agente é um considerado um produto de agentes interpdenententes. Na sua visão, as interdependências das organizações surgem por estas serem um sistma aberto e não são necessariamente simétricas ou balanceadas. Eles resumem a capacidade de uma organização exercer influência sobre outra como sendo um produto do seu controle sobre recursos demandados pela segunda, a dependênca desta em relação aos referidos recursos, sua falta de contrapartidas para equilibrar a relação e sua falta de acesso a recursos alternativos.

Vale notar que há uma predominância do poder coercitivo nos artigos analisados com essa teoria. Isso denota um viés opressor para o estudo do poder com tal lente teórica. A ocorrência de outros tipos de poder simultaneamente estudados juntamente com o poder coercitivo induz questionamentos acerca da sua interação. É de se esperar que os poderes apresentados em uma mesma análise se sustentem sob as mesmas premissas escolhidas pelo pesquisador. Assim, pode-se propor o seguinte:

P1. Havendo dependência de recursos entre agentes em uma rede, há uma tendência que o poder seja exercido de forma coercitiva

P2. A combinação da coerção com outros tipos de poder e domínios de recursos reforça a capacidade de influência do agente coerçor

REAd | Porto Alegre - Edição 83 - N 1 - Janeiro/Abril 2016 - p. 01-25 


\section{INFLUÊNCIA DO PODER NAS REDES DE NEGÓCIO - ANÁLISE DA EVOLUÇÃO DA TEORIA}

\subsubsection{Teoria da Troca em Redes}

Cook et al. (1983) estão especialmente interessados em redes de troca negativamente conectadas, ou seja, aquelas em que há equivalência entre diferentes agentes da rede como fontes de recursos alternativas. Esses autores seguem Emerson (1962), ao considerar o poder entre dois atores como uma função do valor de determinado recurso de um para outro e das possibilidades deste segundo agente obter o mesmo recurso de outras fontes alternativas.

Aqueles autores argumentam que o poder é uma função da posição do agente na rede, ainda que esta seja por ele ignorada. As próprias circunstâncias que cercam o agente conduziriam a uma posição de equilíbrio de poder a partir das condições que se dão as trocas entre os diferentes atores conectados. Sua linha de raciocício chama a atenção para a necessidade de se pensar a rede para além das relações diretas e considerar o poder como um fenômeno que alcança as dimensões da rede.

O desenvolvimento do poder de agentes específicos depende de um conjunto mais amplo de variáveis, portanto, à medida que as trocas se desenvolvem. As regras básicas são conhecidas, mas estados futuros não podem ser completamente previstos - principalmente considerando a visão dos autores de que o poder pode existir como potencial e não ser utilizado em sua totalidade pelos agentes detentores de poder.

Essas características sublinham elementos de cunho subjetivo no raciocínio de Cook et al. (1983). Considerando que essa teoria aparece associada a tantos tipos de poder quando a teoria da dependência de recursos, e que há uma prevalência semelhante do poder coercitivo e a ocorrência deste combinado a outros tipos de poder, propoe-se:

P3. Os agentes utilizam do poder coercitivo como um mecanismo para compensar efeitos negativos da dinâmica da rede sobre sua posição de poder

P4. A combinação da coerção com outros tipos de poder reforça a capacidade do agente coerçor anular efeitos negativos da dinâmica da rede sobre sua posição de poder 


\subsubsection{Teoria de Custos de Transação}

Williamson (1981) apresenta a teoria de custos de transação interpretando as decisões estruturais sobre os relacionamentos de uma rede como decisões estratégicas relacionadas às escolhas entre economias de economias de produção e economias de transação. Relacionando como as dimensões críticas de uma transação sua incerteza, sua frequência e o grau de especificidade dos investimentos, o autor observa que esta última é a principal delas e correspontem aos recursos tecnológicos essenciais.

A teoria de custos de transação coloca o agente em uma perspectiva de tomador de decisões racionais. Não há um tipo de poder em particular com uma recorrencia de destaque, como no caso da teoria de dependência de recursos. Entretanto, é interessante notar a ausência do poder de recompensa, presente nas outras teorias mais frequentes. Esse tipo de poder representa a visão de que a capacidade de conferir recompensas é um instrumento de poder na rede.

Ainda, o fato de esse tipo de poder se fundamentar em um tipo específico de recursos - os recursos tecnológicos - denota seu aspecto objetivista. A decisão de internalizar ou não uma transação é mensurável de maneira concreta. A respeito disso, pode-se propor:

P5. A recompensa, como veículo de poder, não afeta a incerteza de uma transação entre agentes em uma rede.

P6. A recompensa, como veículo de poder, não afeta a frequência de uma transação entre agentes em uma rede.

P7. A recompensa, como veículo de poder, não afeta o grau de especificidade de investimentos de uma transação entre agentes em uma rede.

\section{CONSIDERAÇÕES FINAIS}

Com a revisão sistemática realizada, esta pesquisa buscou compreender como as relações de poder são abordadas na literatura de organizações, utilizando a base de dados Thomson Reuters (ISI) Web of Science. Além disso, também se refletiu sobre as implicações dos paradigmas privilegiados na literatura pesquisada, por meio da construção de proposições conceituais relacionando tais paradigmas à tipologia tradicional de poder. Conforme indicado anteriormente, a existência perspectivas preferenciais indica premissas e limites de validade 


\section{INFLUÊNCIA DO PODER NAS REDES DE NEGÓCIO - ANÁLISE DA EVOLUÇÃO} DA TEORIA

particulares no estudo de um fenômeno (MORGAN, 1980). A discussão realizada procurou identificar esse viés no campo selecionado, com base nos artigos filtrados na busca.

A multiplicidade de abordagens existentes para a discussão de poder reforça o caráter dinâmico de uma área que está em expansão. Do ponto de vista do delineamento da pesquisa, a presença dos estudos de caso é expressiva, como esperado (FREESE, 1980; TODEVA, 2006), mas outros delineamentos aparecem - com destaque para pesquisas bibliográficas. A respeito das lentes teóricas utilizadas, os artigos refletem a divisão do campo com respeito a diferentes linhas conceituais. A teoria da dependência de recursos ganha destaque no quadro geral, contudo, o que remete aos principais paradigmas de estudo de redes.

O resultado não é estranho, dado que as abordagens relacional e estrutural, principais paradigmas no estudo das redes de negócios, podem ser ancoradas nessa referência. A proeminância dessa perspectiva ficou evidente também na análise do mapa de cocitações dos artigos pesquisados. Adicionalmente, a disputa com outras publicações pelo destaque nos rankings de centralidade e intermediação do mapa de cocitações indica a trajetória de atualização conceitual do campo. Por fim, a distribuição dos artigos segundo os diferentes tipos de poder definidos na literatura serviu de insumo, juntamente com as principais teorias identificadas (i.e., Dependência de Recursos, Troca em Redes e Custos de Transação), para o delineamento de proposições para investigações futuras.

São limitações particulares deste estudo o horizonte de tempo considerado para a seleção dos textos avaliados e o caráter interpretativo da classificação dos artigos em função dos tipos de poder recuperados da literatura. Estudos futuros que investiguem e validem as proposições apresentadas contribuirão para o avanço da discussão aqui iniciada com o aprofundamento da compreensão sobre o poder em redes de negócios.

\section{REFERÊNCIAS}

BELAYA, V.; HANF, J. H. Managing Russian agri-food supply chain networks with power. Journal on Chain and Network Science, v. 12, n. 3, p. 215-230, 2012.

BENGTSSON, M.; KOCK, S. "Coopetition" in Business Networks - to Cooperate and Compete Simultaneously. Industrial Marketing Management, v. 29, n. 5, p. 411-426, 2000.

BIERSTEDT, R. An Analysis of Social Power. American Sociological Review, v. 15, n. 6, REAd | Porto Alegre - Edição 83 - No 1 - Janeiro/Abril 2016 - p. 01-25 
p. $730-738,1950$.

BLOIS, K. The legitimacy of power in business-to-business relationships. Marketing Theory, v. 10, n. 2, p. 161-172, 2010.

BONOMA, T. V.; JOHNSTON, W. J. The social psychology of industrial buying and selling. Industrial Marketing Management, v. 7, n. 4, p. 213-224, 1978.

CALDAS, M. P. Paradigmas em Estudos Organizacionais: Uma introdução à Série. Revista de Administração de Empresas, v. 45, n. 1, p. 53-57, 2005.

CASCIARO, T. Determinants of governance structure in alliances: the role of strategic, task and partner uncertainties. Industrial and Corporate Change, v. 12, n. 6, p. 1223-1251, 2003.

CHENG, T.-K.; SCULLI, D.; CHAN, F. S.-F. Relationship dominance. Journal of Managerial Psychology, v. 16, n. 2, p. 97-105, 2001.

COOK, K. S. et al. The Distribution of Power in Exchange Networks: Theory and Experimental Results. American Journal of Sociology, v. 89, n. 2, p. 275-305, 1983.

COX, A. et al. Managing appropriately in power regimes: Relationship and performance management in 12 supply chain cases. Supply Chain Management, v. 9, n. 5, p. 357-371, 2004.

DAHL, R. A. The Concept of Power. Behavioral Science, v. 2, n. 3, p. 201-215, 1957.

DENYER, D.; TRANFIELD, D. Producing a Systematic Review. In: BUCHANAN, D.; BRYMAN, A. (Eds.). . The SAGE Handbook of Organizational Research Methods. [s.1.] SAGE Publications, 2009. p. 671-689.

DYER, J. H.; SINGH, H. The Relational View: Cooperative Strategy and Sources of Interorganizational Competitive Advantage. The Academy of Management Review, v. 23, n. 4, p. 660-679, 1998.

EMERSON, R. M. Power-Dependence Relations. American Sociological Review, v. 27, n. 1, p. 31-41, 1962.

FLIGSTEIN, N. Habilidade social e a teoria dos campos. Revista de Administração de Empresas, v. 47, n. 2, p. 61-80, 2007. 
INFLUÊNCIA DO PODER NAS REDES DE NEGÓCIO - ANÁLISE DA EVOLUÇÃO DA TEORIA

FREESE, L. Formal Theorizing. American Review of Sociology, v. 6, n. 1, p. 187-212, 1980.

FRENCH, J. R. P.; RAVEN, B. The Bases of Social Power. In: Studies in Social Power. Ann Arbor, MI: University of Michigan Press, 1959. p. 150-167.

GRANOVETTER, M. S. The Strength of Weak Ties. American Journal of Sociology, v. 78, n. 6, p. 1360-1380, 1973.

GULATI, R.; NOHRIA, N.; ZAHEER, A. Strategic Networks. Strategic Management Journal, v. 21, n. 3, p. 203-215, 2000.

HÅKANSSON, H.; FORD, D. How should companies interact in business networks? Journal of Busines Research, v. 55, n. 2, p. 133-139, 2002.

HÅKANSSON, H.; SNEHOTA, I. (EDS.). Developing Relationships in Business Networks. London and New York: Routledge, 1995.

HANDLEY, S. M.; BENTON, W. C. The influence of exchange hazards and power on opportunism in outsourcing relationships. Journal of Operations Management, v. 30, n. 12, p. 55-68, 2012.

HINGLEY, M. K. Power to all our friends? Living with imbalance in supplier-retailer relationships. Industrial Marketing Management, v. 34, n. 8, p. 848-858, 2005.

HO, D. Y. F. Relational Orientation and Methodological Individualism. Bulletin of the Hong Kong Psychological Society, v. 26/27, p. 81-95, 1991.

JENSEN, M. C.; MECKLING, W. H. Theory of the Firm: Managerial Behavior, Agency Costs and Ownership. Journal of Financial Economics, v. 3, n. 4, p. 305-360, 1976.

LEWIS, M. W.; GRIMES, A. J. Metatriangulation: Building Theory from Multiple Paradigms. The Academy of Management Review, v. 24, n. 4, p. 672, 1999.

MEDCOF, J. W. Resource-based strategy and managerial power in networks of internationally dispersed technology units. Strategic Management Journal, v. 22, n. 11, p. 999-1012, 2001.

MEEHAN, J.; WRIGHT, G. H. The origins of power in buyer-seller relationships. Industrial REAd | Porto Alegre - Edição 83 - N 1 - Janeiro/Abril 2016 - p. 01-25 
Marketing Management, v. 41, n. 4, p. 669-679, 2012.

MORGAN, G. Paradigms, Metaphors, and Puzzle Solving in Organization Theory. Administrative Science Quarterly, v. 25, n. 4, p. 605-622, 1980.

PFEFFER, J.; SALANCIK, G. R. Social Control of Organizations. In: The External Control of Organizations: A Resource Dependence Perspective. New York, NY: Harper \& Row, 1978. p. 39-61.

RICCOBONO, F.; BRUCCOLERI, M.; PERRONE, G. Business agreements objectives and decisions: a field research. Management Research Review, v. 36, n. 5, p. 495-527, 2013.

RODRIGUEZ, C. et al. Governance, Power, and Mandated Collaboration in an Interorganizational Network. Administration \& Society, v. 39, n. 2, p. 150-193, 2007.

SANDERSON, J. Opportunity and constraint in business-to-business relationships: insights from strategic choice and zones of manoeuvre. Supply Chain Management, v. 9, n. 5, p. 392-401, 2004.

SMITH-DOERR, L.; POWELL, W. W. Networks and Economic Life. In: The Handbook of Economic Sociology. Princeton: Princeton University Press, 2004. p. 368-402.

TODEVA, E. Business Networks: Strategy and Structure. London: Routledge, 2006.

TRANFIELD, D.; DENYER, D.; SMART, P. Towards a Methodology for Developing Evidence-Informed Management Knowledge by Means of Systematic Review. British Journal of Management, v. 14, n. 3, p. 207-222, 2003.

WEBSTER, E. General Model for Understanding Organization Buying Behavior. The Journal of Marketing, v. 36, n. 2, p. 12-19, 1972.

WILLIAMSON, O. E. The Economics of Organization: The Transaction Cost Approach. The American Journal of Sociology, v. 87, n. 3, p. 548-577, 1981.

ZACCARELli, S. B. et al. Clusters e Redes de Negócios. São Paulo: Atlas, 2008. 